\title{
Functional Asymmetry of Human Prefrontal Cortex: Encoding and Retrieval of Verbally and Nonverbally Coded Information
}

\author{
Bertram Opitz, ${ }^{1}$ Axel Mecklinger, and Angela D. Friederici \\ Max-Planck-Institute of Cognitive Neuroscience, Leipzig, D-04103 Germany
}

\begin{abstract}
There are several views about the organization of memory functions in the human prefrontal cortex. One view assumes a process-specific brain lateralization according to different memory subprocesses, that is, encoding and retrieval. An alternative view emphasizes content-specific lateralization of brain systems involved in memory processes. This study addresses this apparent inconsistency between process- and content-specific lateralization of brain activity by investigating the effects of verbal and nonverbal encoding on prefrontal activations during encoding and retrieval of environmental novel sounds using fMRI. An intentional memory task was applied in which subjects were required either to judge the sounds' loudness (nonverbal encoding task) or to indicate whether or not a sound can be verbally described (verbal encoding task). Retrieval processes were examined in a subsequent yes/no recognition test. In the study phase the right posterior dorsolateral prefrontal cortex (PFC) was activated in both tasks. During verbal encoding additional activation of the left dorsolateral PFC was obtained. Retrieval-related fMRI activity varied as a function of encoding task: For the nonverbal task we detected an activation focus in the right posterior dorsolateral PFC whereas an activation in the left dorsolateral PFC was observed for the verbal task. These findings indicate that the right dorsolateral PFC is engaged in encoding of auditory information irrespective of encoding task. The lateralization of PFC activity during retrieval was shown to depend on the availability of verbal codes, with left hemispheric involvement for verbally and right hemispheric activation for nonverbally coded information.
\end{abstract}

Human electrophysiological, neuropsychological, and functional imaging studies have highlighted the importance of frontal brain regions in memory (Pigott and Milner 1993; Tulving et al. 1994; Wilding and Rugg 1996). However, the functional significance of these regions for mnemonic processes is a matter of an ongoing debate (Kelley 1998a; $\mathrm{Ny}$ berg et al. 1998). The activation patterns reported in the literature seem to support both process- and material-specific involvement of frontal cortex in episodic memory.

A process-specific lateralization of frontal brain regions has been suggested by Tulving and colleagues (Nyberg et al. 1996; Tulving et al. 1994). They proposed a hemispheric encoding/retrieval asymmetry (HERA) model, claiming that left frontal lobes are more involved in episodic memory encoding whereas right frontal lobes are recruited by retrieval from episodic memory. Those neuroimaging studies observing results consistent with HERA mostly investigated either encoding or retrieval of verbal stimuli (words).

A material-specific lateralization of brain systems in memory processes was proposed initially on the basis of neuropsychological studies (Milner and Taylor 1972; Gazzaniga and Smylie 1983). This view was confirmed by recent

${ }^{1}$ Corresponding author.

E-MAIL opitz@cns.mpg.de; Fax 493419940113. neuroimaging studies investigating memory encoding (Brewer et al. 1998; Kelley et al. 1998b; Wagner et al. 1998b,c). Two of these studies (Brewer et al. 1998; Wagner et al. 1998c) used a similar paradigm sorting items in the study phase based on whether they were subsequently remembered or not. The two studies, however, differed in the stimulus material examined. Using verbal materials (words), Wagner and colleagues (1998c) obtained left frontal activation during successful memory encoding, whereas Brewer et al. (1998) used nonverbal materials (visual scenes) and found encoding-specific activation in the right frontal cortex. A similar right lateralized brain activity was reported in a recent functional imaging study examining the encoding of nonfigurative pictures (Klingenberg and Roland 1998).

Material-specific lateralization was also demonstrated when directly comparing the encoding of verbal materials with the encoding of nonverbal materials in the same subjects (Kelley et al. 1998b; Wagner et al. 1998b; McDermott et al. 1999). This finding was explained by differences in the amount of verbal processing encouraged by the different materials. That is, words tend to elicit verbal processing and thereby left-lateralized brain activity whereas faces or textures elicit nonverbal processing associated with right-lateralized prefrontal activation.

Despite converging evidence for material-specific lat-

LEARNING \& MEMORY 7:85-96 @ 2000 by Cold Spring Harbor Laboratory Press ISSN1072-0502/00 \$5.00

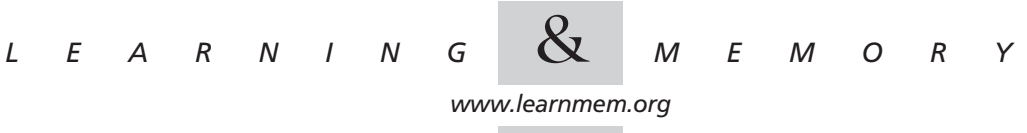


eralization, other studies observed bilateral or even left-lateralized brain activity during encoding of nonverbal material such as unfamiliar faces (Haxby et al. 1995, 1996) or visual objects (Kelley et al. 1998b; A. Mecklinger, V. Bosch, C. Grünewald, S. Bentin, and D.V. von Cramon, in prep.). One possible explanation is that differences in the complexity of the verbal and nonverbal material cause differential involvement of frontal cortex during memory encoding. Alternatively, the contributions of specific prefrontal regions to memory encoding might depend on different aspects of verbal materials, such as phonological, lexical, or semantic aspects (see also Wagner et al. 1998c for a similar argument). There is recent evidence that semantic aspects indeed play an important role in processing nonverbal material (Mecklinger et al. 1997; W.M. Kelley, R.L. Buckner, F.M. Miezin, N.J. Cohen, M.E. Raichle, and S.E. Petersen, unpubl.). W.M. Kelly, R.L. Buckner, F.M. Miezin, N.J. Cohen, M.E. Raichle, and S.E. Peterson (unpubl.) showed that the left prefrontal cortex is activated during the encoding of famous faces, for which names are easily accessible. Thus, prefrontal engagement in episodic memory might be determined by the availability of lexical-semantic codes for verbal and nonverbal materials (content-specific lateralization) rather than by the distinction between verbal and nonverbal material per se (material-specific lateralization).

This study tests the hypothesis of a content-specific lateralization of prefrontal regions versus the process-specific view by using a two-by-two factorial design varying the content of the material (verbal, nonverbal) and the process required (encoding, retrieval). In contrast to previous visual studies (Kelley et al. 1998b; Wagner et al. 1998b; McDermott et al. 1999), we manipulated the stimulus content, that is, the availability of verbal codes, within the same class of nonverbal stimuli, namely environmental novel sounds. This allows us to extend the above-mentioned findings from visual studies to the auditory stimulus domain, providing a basis for a possible generalization across input domains. In a prior study (Mecklinger et al. 1997) we examined eventrelated brain potentials (ERPs) evoked by environmental sounds that differ in the availability of a semantic concept (identifiable and nonidentifiable sounds) in auditory classification tasks. This study revealed that identifiable in contrast to nonidentifiable sounds showed a N400 component-a component usually found in correlation with lexical-semantic integration processes (Kutas and Hillyard 1983). It was suggested that this $\mathrm{N} 400$ to sounds reflects the access of semantic content expressed by identifiable sounds. In this study we used the same kind of stimuli, that is, environmental sounds, in an explicit memory task under different task instructions. Task instructions focusing on either nonverbal or verbal features of the stimuli were employed to control for the nature of codes subjects invoke for memorizing these sounds. Because verbal as well as nonverbal codes are available for identifiable sounds, explicit task instructions would emphasize the use of either of the two codes.

The content-specific view, which holds that the lateralization of prefrontal memory functions is dependent on the availability of verbal codes, would predict primarily left prefrontal activity for the verbal task and right prefrontal activity for the nonverbal task. Furthermore, the left hemispheric brain activity should be modulated by the intrinsic verbal/nonverbal features of sounds, that is, a greater left prefrontal activity for identifiable as compared to nonidentifiable sounds.

\section{RESULTS}

\section{Behavioral Results}

Subjects correctly recognized $67.5 \%$ of the old items across conditions. Mean probability of a hit minus probability of a false alarm $(\mathrm{Pr})$ was 0.14 . This value was significantly different from zero $(P<0.05)$ for each of the four conditions (Table 1), suggesting that recognition performance was above chance in both tasks and for both enviromental sound (hereafter referred to as novel) types. A possible account for this low recognition level might be changes in accuracy during the relative long recognition period. This hypothesis was tested in a post hoc analysis by contrasting performance during the first and the second half of the recognition period for both novel types. Neither the main effects of task and period of analysis nor their interaction were significant (all $P$ values $>0.6$ ).

Although identifiable novels (Pr 0.146 and 0.166) were slightly better recognized than nonidentifiable novels ( $\mathrm{Pr}$ 0.113 and 0.109 ) in both encoding conditions (Table 1), this difference in recognition performance did not reach significance. Neither the main effects encoding task $\left(F_{1,16}<1\right)$ and novel type $\left(F_{1,16}=3.06 ; P<0.1\right)$ nor the interaction between the two factors $\left(F_{1,16}<1\right)$ were significant in an ANOVA contrasting the Pr values for the two novel types in both tasks.

\section{Imaging Results \\ Encoding Phase}

A number of brain areas showed blood-oxygen level dependent (BOLD) signal increases (activation) in the encoding

\begin{tabular}{|c|c|c|c|c|}
\hline & \multicolumn{2}{|c|}{ Identifiable novels } & \multicolumn{2}{|c|}{ Nonidentifiable novels } \\
\hline & $\begin{array}{l}\text { Verbal } \\
\text { task }\end{array}$ & $\begin{array}{c}\text { Nonverbal } \\
\text { task }\end{array}$ & $\begin{array}{l}\text { Verbal } \\
\text { task }\end{array}$ & $\begin{array}{c}\text { Nonverbal } \\
\text { task }\end{array}$ \\
\hline Mean & 0.146 & 0.166 & 0.113 & 0.109 \\
\hline S.E.M. & 0.049 & 0.060 & 0.030 & 0.028 \\
\hline
\end{tabular}

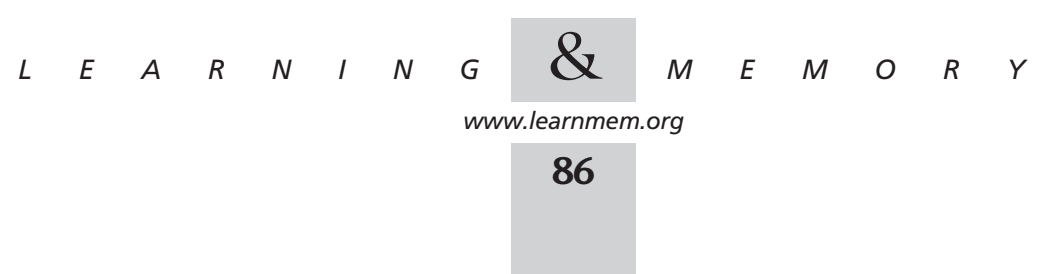


and recognition phases, regardless of novel type and encoding task. These areas included the posterior part of the left inferior frontal sulcus near Brodmann area (BA) 44/9 with homologous activation in the right hemisphere. Similar areas were found previously to be active during the encoding of words (Buckner et al. 1998a; Kelley et al. 1998b) and visual objects (Kelley et al. 1998b) and were labeled as dorsolateral prefrontal cortex (DLPFC). In the following we will refer to this activation of BA 44/9 as DLPFC activation. Further, the bilateral anterior insular cortex near the frontal operculum, as well as associated thalamic structures (Table 2 ), was consistently activated. These activations were similar to activations previously observed in episodic memory tasks (Buckner et al. 1998b).

To investigate the activation in these regions for each of the conditions, the BOLD response of these regions was first compared across the verbal and nonverbal encoding tasks. Figures 1 and 2 show significant dorsal frontal activation for both stimuli in each of the encoding tasks. In the nonverbal task (Fig. 1) significant brain activation was observed in the right posterior DLPFC. In the verbal task (Fig. 2) an additional activation of the left DLPFC was obtained. These activations of the left and right posterior DLPFC exhibited significant differences as a function of encoding task and novel type.

The mean activation values of spherical regions defined around the peak activations (Fig. 3) entered an ANOVA with the between subject factor encoding task (nonverbal/verbal) and the within-subject factors novel type (identifiable/ nonidentifiable) and hemisphere (left/right). This analysis revealed a significant main effect of novel type $\left(F_{1,16}=9.91\right.$, $P<0.01)$, with greater activation for identifiable than for nonidentifiable novels.

Additionally, a significant encoding task by hemisphere interaction $\left(F_{1,16}=5.21, P<0.05\right)$ indicated that the nonverbal task produced greater right than left dorsolateral prefrontal activation, whereas a more bilateral activation was found in the verbal task. Post hoc tests performed separately for each of the encoding tasks confirmed the finding of a lateralized brain activity in the nonverbal task (main effect hemisphere, $F_{1,8}=4.67, P<0.06$ ), but not in the verbal task $\left(F_{1,8}=2.38, P<0.3\right)$. This dissimilar lateralization was due to differences in left hemispheric prefrontal activation, with greater involvement of this brain structure in the verbal task as compared to the nonverbal task (main effect of encoding task within the left hemisphere $\left.F_{1,16}=5.21, P<0.05\right)$. Conversely, the right DLPFC was not differentially activated by the two encoding tasks $\left(F_{1,16}<1\right)$. A marginally significant triple interaction between encoding task, novel type, and hemisphere $\left(F_{1,16}=4.26 ; P<0.06\right)$ suggests that in the nonverbal task identifiable novels exhibited greater left dorsofrontal brain activity than nonidentifiable novels, whereas in the verbal task no left hemispheric difference as a function of novel type was obtained.

In summary, encoding of novel sounds was associated with an increased BOLD response in the right DLPFC irrespective of novel type and encoding task. Conversely, in the left DLPFC encoding-related brain activity was higher for identifiable than for nonidentifiable novels. This pattern of activity was modulated by the task factor: In the nonverbal task identifiable novels exhibited greater activity in the left prefrontal cortex (PFC) than did nonidentifiable novels.

Other notable regions exhibiting significant BOLD responses during the encoding of novel sounds included the anterior insula/frontal operculum, the superior temporal plane, and the thalamus. Table 3 summarizes these activations, all of them located bilaterally for identifiable and nonidentifiable novels in both encoding tasks. The same ANOVA as for the DLPFC was applied to these regions. There were no significant differences of brain activity across hemispheres as well as no significant effects of encoding task or novel type (all $F$ values $<1$ ). Although these findings indicate that various cortical and subcortical brain structures support the processing of novel sounds, their activation might not be related to the encoding of these sounds into memory. It is possible that these brain activations represent memory unspecific processes associated

Table 2. Identification of BOLD Signal Increases in Encoding and Recognition of Novel Sounds

\begin{tabular}{|c|c|c|c|c|c|c|c|c|}
\hline \multirow[b]{2}{*}{ Location } & \multicolumn{4}{|c|}{ Encoding (coordinates) } & \multicolumn{4}{|c|}{ Recognition (Coordinates) } \\
\hline & $x$ & Y & $Z$ & $z$-value & $x$ & Y & $Z$ & $z$-value \\
\hline Left posterior DLPFC & -46 & 8 & 29 & 4.48 & -46 & 6 & 33 & 3.31 \\
\hline Right posterior DLPFC & 45 & 8 & 25 & 3.99 & 45 & 9 & 30 & 3.87 \\
\hline Left anterior insula & -37 & 21 & 13 & 5.40 & -33 & 19 & 7 & 3.43 \\
\hline Right anterior insula & 38 & 24 & 12 & 6.11 & 30 & 19 & 7 & 3.01 \\
\hline Left sup. temporal plane & -51 & -26 & 15 & 6.75 & & & - & \\
\hline Right sup. temporal plane & 51 & -24 & 13 & 5.58 & & & - & \\
\hline Left thalamus & -12 & -18 & 12 & 6.54 & -14 & -19 & 11 & 4.40 \\
\hline Right thalamus & 6 & -15 & 12 & 6.79 & 6 & -16 & 12 & 4.20 \\
\hline
\end{tabular}

Coordinates are listed in Talairach and Tournoux (1988).

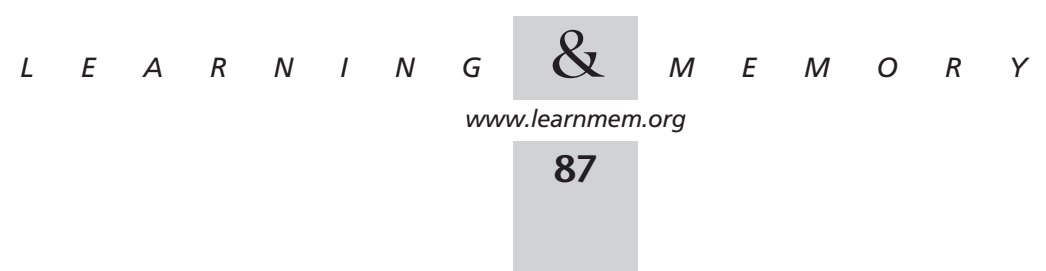




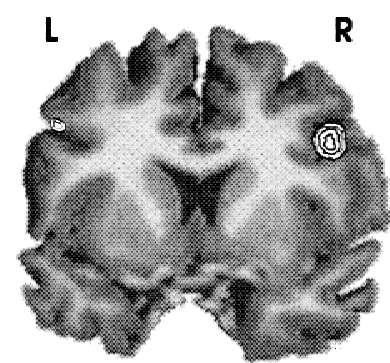

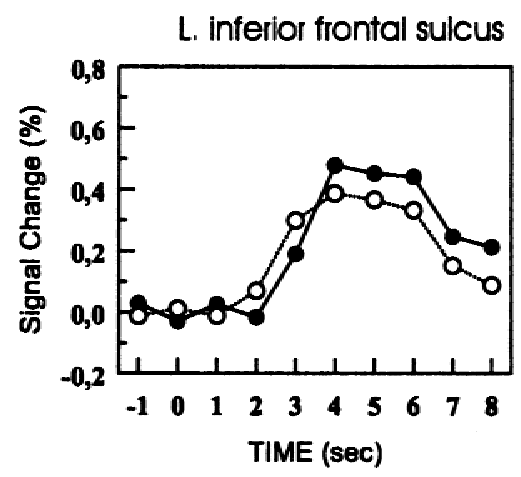

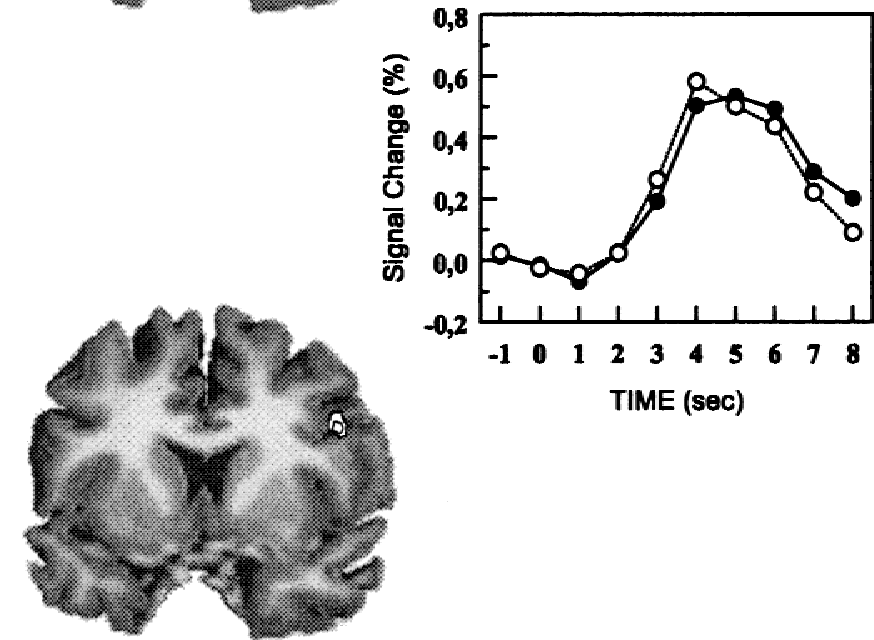

\section{R. inferior frontal sulcus}

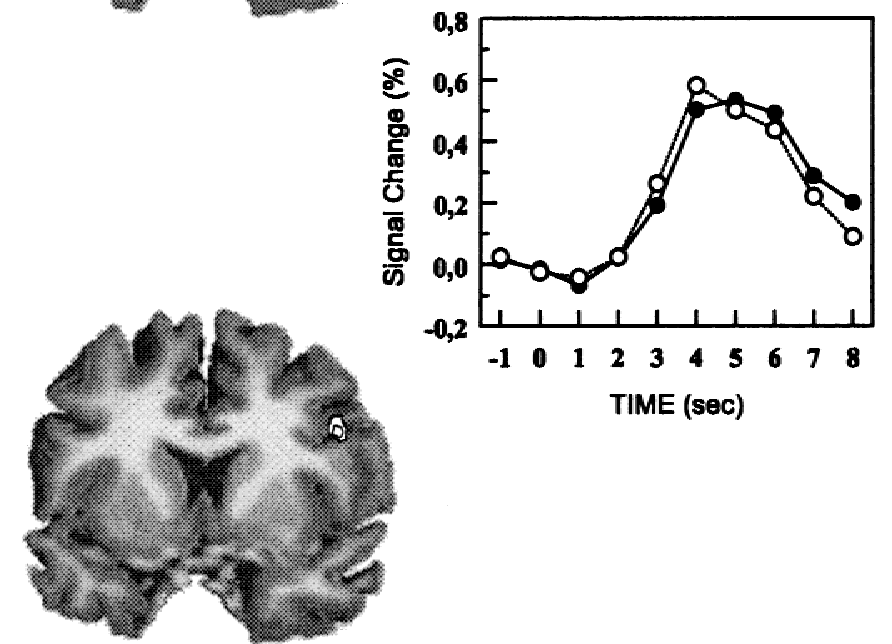

O non identifiable sounds

Figure 1 Encoding phase nonverbal task. Dorsolateral prefrontal cortex activation for identifiable (top, $\mathbf{0})$ and nonidentifiable $(b o t t o m, ~ O)$ novel sounds. The coronal sections show significant activations averaged across subjects. Functional images were superimposed on an individual brain in Talairach space. The images were thresholded at $z=3.09(P<0.001$, one tailed). Contour lines illustrate regions of equal $z$-scores and are plotted in increments of $0.5 z$. Peak activations were located in right $(40,10,30$ and $42,12,26)$ dorsolateral prefrontal cortex for identifiable and nonidentifiable novels, respectively. The left and right panels present the time course plots of peak activation for both novel types for the left and right hemispheres, respectively.

with the verbal and nonverbal judgments to be made (Buckner et al. 1996b).

To examine which of the aforementioned activation pattern contributed to subsequent memory performance a second analysis was performed. This analysis was based on a Kendall's rank-order correlation between the encodingrelated brain activity to the novel sounds and subsequent recognition performance. This subsequent memory effect was rather specific to the PFC: Whereas the activity of the left and right DLPFC was predictive with respect to whether sounds would be remembered or forgotten (Table 4), other regions active during novel processing failed to demonstrate a systematic relationship between brain activity during encoding and subsequent memory performance.

In the verbal task left posterior DLPFC activation was significantly correlated with the recognition performance for identifiable novel sounds $(r=0.59)$. Conversely, in the nonverbal task a marginally significant correlation between right dorsolateral prefrontal brain activity during encoding and recognition performance for identifiable novels was obtained $(r=0.50)$. In addition, in the nonverbal task the activity of the left DLPFC seemed to be negatively corre- lated with memory performance for nonidentifiable novels $(r=-0.54)$.

\section{Recognition Phase}

In the recognition phase correctly remembered sounds compared to pure tones activated several brain regions that overlapped considerably with those found to be activated in the encoding phase (Table 2). These regions included lateralized DLPFC and the anterior insula and the thalamus bilaterally.

An ANOVA examining the effects of encoding task, novel type, and hemisphere separately for each of these regions revealed significant effects for the DLPFC only (Figs. 4 and 5). For this region a hemisphere $x$ encoding task interaction was observed $\left(F_{1,16}=8.5, P<0.01\right)$. Post hoc tests performed on the mean $z$-scores (Fig. 6) separately for each of the two encoding tasks revealed left lateralized brain activity in the verbal task (main effect hemisphere, $\left.F_{1,8}=11.2, P<0.01\right)$. Moreover, in the left hemisphere greater activity was obtained in the verbal task as compared to the nonverbal task $\left(F_{1,8}=5.75, P<0.05\right)$. In this latter task we observed for both novel types a significant activa-

$$
\begin{array}{lllllllllllllll}
L & E & A & R & N & I & N & G & \mathcal{Q} & M & E & M & O & R & Y \\
\text { www.learnmem.org } & &
\end{array}
$$




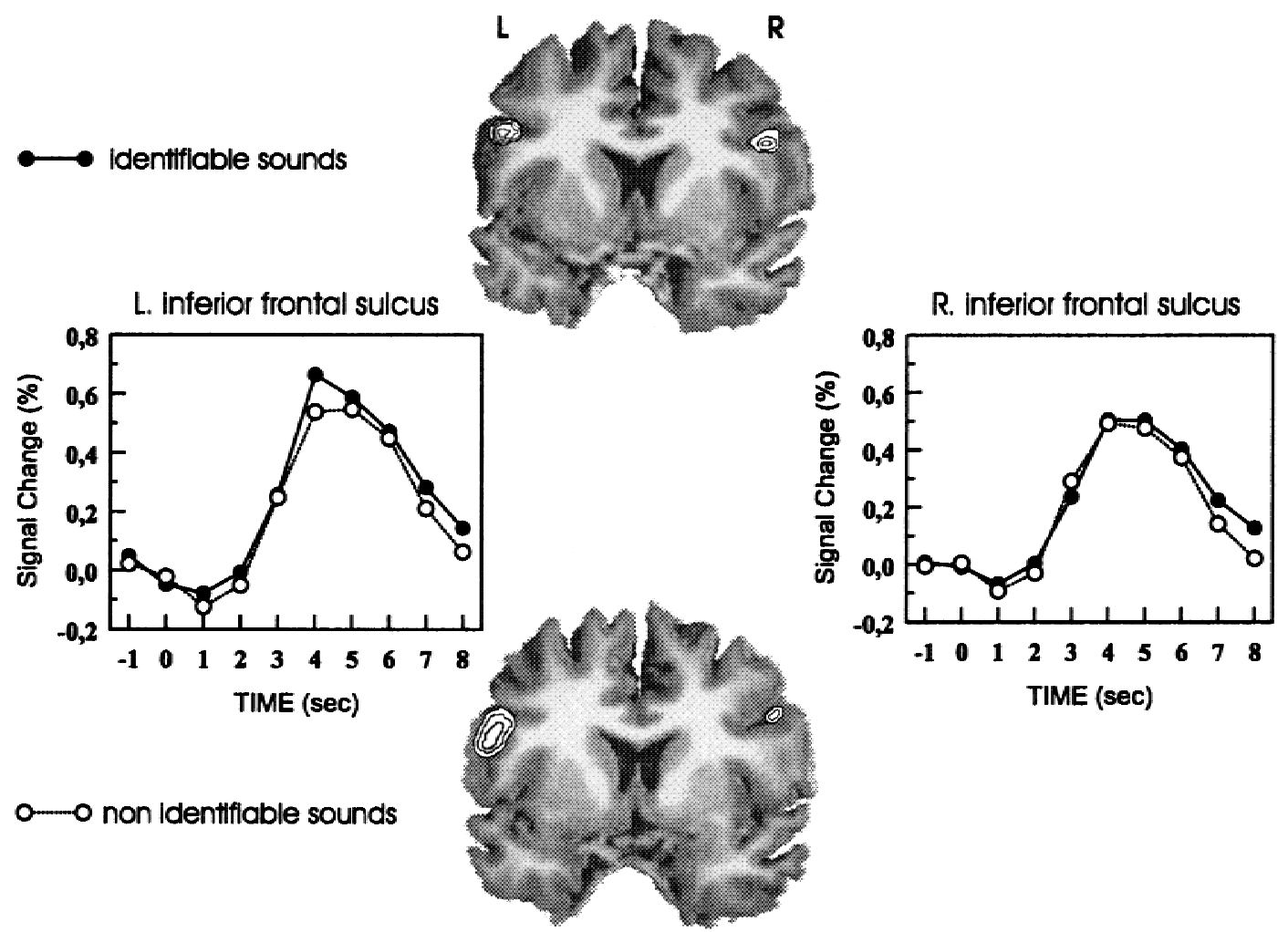

Figure 2 Encoding phase verbal task. Dorsolateral prefrontal cortex activation for identifiable (top, $\bullet$ ) and nonidentifiable (bottom, $\bigcirc)$ novel sounds. Peak activations were located in left $(-46,14,27)$ and right $(43,14,23)$ dorsolateral prefrontal cortex for identifiable novels. Nonidentifiable novels activated the left $(-50,6,20)$ dorsolateral prefrontal cortex and the right $(42,12,26)$ dorsolateral prefrontal cortex. (For details, see Fig. 1.)

tion in the right DLPFC but not in the left DLPFC. Despite this, there was no main effect of hemisphere $\left(F_{1,8}=1.3\right.$, $P<0.2)$ in an ANOVA performed on the mean $z$-scores.

In contrast to the prediction of a stronger right hemispheric involvement in episodic retrieval based on HERA the present data suggest less activity during recognition as compared to encoding. To test this specific hypothesis a three-way ANOVA additionally including a factor process (encoding, retrieval) was performed on the $z$-scores of a right dorsolateral prefrontal region identified in the data collapsed across encoding and retrieval scans. A main effect of process $\left(F_{1,16}=5.64, P<0.05\right)$ indicated greater involvement of the right DLPFC during encoding than during recognition.

As during encoding no significant influences of the encoding task or novel type on insular and thalamic brain activity during recognition were observed, thereby confirming the view of a memory unspecific contribution of these brain regions (Table 5).

\section{DISCUSSION}

In this study we used two encoding tasks emphasizing either the processing of verbal or the processing of physical aspects of novel sounds to assess content-specific hemispheric specialization of the PFC in the encoding and recognition phases of an intentional memory task. The PFC exhibited a similar activation pattern during encoding and recognition with left-lateralized brain activity in a verbal encoding task and a right-lateralized activation in a nonverbal encoding task. Furthermore, these brain activations were modulated by the identifiably of the novel sounds

\section{Activation in the Dorsolateral Prefrontal Cortex} Equivalent right hemispheric activation in the posterior DLPFC was observed during encoding for identifiable and nonidentifiable novel sounds in the two tasks. This activity might reflect nonverbal stimulus encoding based on the physical features of a particular stimulus. Consistent with this result, several neuroimaging studies report right dorsal frontal activations during tasks that encourage encoding of nonverbal material in the visual domain, such as pictures of faces and scenes (Brewer et al. 1998; Kelley et al. 1998b; Klingenberg and Roland 1998) or in the auditory domain (Zatorre et al. 1994). Furthermore, an additional left dorsolateral prefrontal activation was observed for both novel types during verbal encoding, possibly reflecting the at-

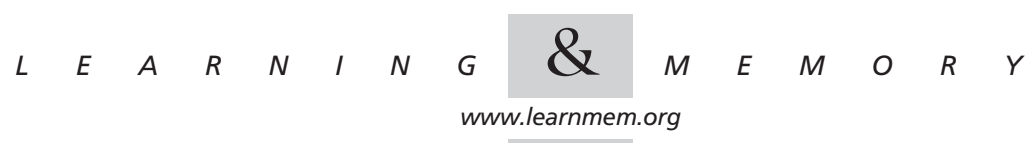



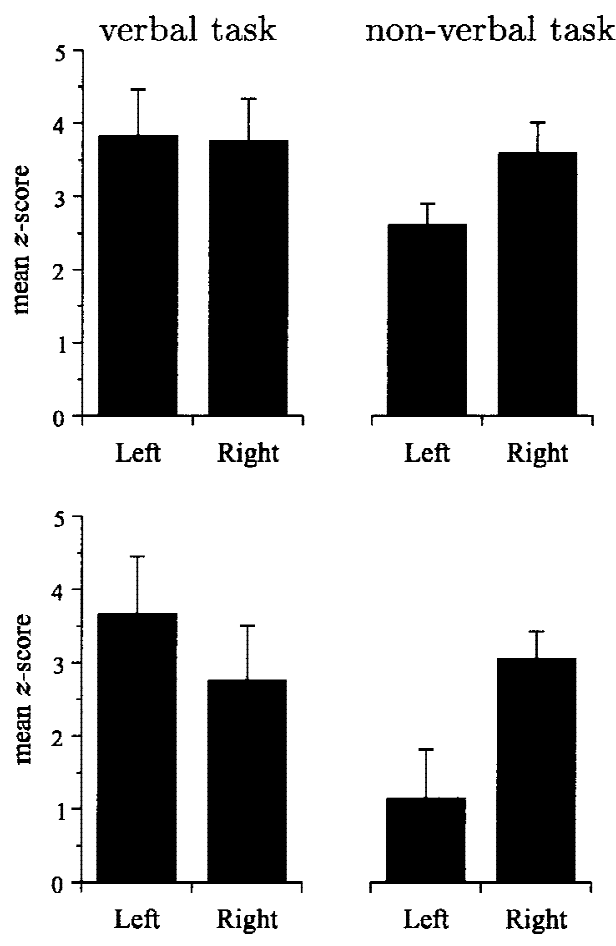

Figure 3 Encoding phase. Mean activation $z$-values and S.E.Ms of the regions depicted in Figs. 1 and 2. (Top) Identifiable novels; (Bottom) nonidentifiable novels.

tempt of accessing verbal codes for these sounds. Left prefrontal activation evoked by encoding of verbal materials has been consistently described in numerous memory studies across a variety of tasks (Tulving et al. 1994; Dolan and Fletcher 1997; Wagner et al. 1998c). Further evidence for the view that verbal codes are efficiently used for memorizing novel sounds comes from the observation of a positive correlation of left hemispheric brain activity with memory performance within the verbal encoding task. The view that verbal codes, in addition to nonverbal codes were used during encoding is also supported in particular by the finding of additional left dorsolateral brain activity evoked by identifiable but not by nonidentifiable novel sounds in the nonverbal encoding task. Such availability of dual codes might force bilateral prefrontal processing, as revealed by the present and previous data (Kelley et al. 1998b). Thus, the processing of physical features (nonverbal encoding), as reflected in right posterior dorsolateral prefrontal brain activity, seems to be a prerequisite for sound encoding. The availability of verbal codes, reflected in left hemispheric brain activation, might be modulated by both intrinsic stimulus properties (identifiable vs. nonidentifiable sounds) and/or the instructions to verbally or nonverbally encode the stimulus material.

The similarity of the activation pattern during encoding and recognition observed in this study, seems to be inconsistent with the HERA model (Nyberg et al. 1996). This model, in its current form, suggests a preferential involvement of left frontal regions in memory encoding and of right frontal areas in episodic memory retrieval, irrespective of the type of information to be processed. Given that the HERA model was originally based on a review of imaging studies that mainly examined encoding of verbal materials (Tulving et al. 1994), the encoding aspect of the HERA model may hold for verbally coded but not for nonverbally coded information. The encoding of the latter information type rather appears to recruit the right posterior DLPFC.

The recognition of novel sounds in the verbal condition was associated with a left-lateralized activation of the posterior DLPFC. This is in agreement with recent neuroimaging studies that report similar activation for correctly recognized verbal stimuli (Buckner et al. 1998a,b; Henson et al. 1999). Moreover, the lack of equivalent right hemispheric activity in the verbal task indicates that only the verbal code was accessed and retrieved during recognition. A different pattern emerged in the recognition phase of the nonverbal task. In this task correctly recognized novel sounds evoked a significant hemodynamic brain response in the right DLPFC. This might indicate that mainly nonverbal codes were used for recognizing the sounds in the nonverbal task, even though verbal codes were available for identifiable novels during encoding.

For memory retrieval the HERA model claims a right hemispheric dominance. The present results challenge this hypothesis, as they reveal a clear involvement of the left PFC during recognition in the verbal task and a stronger involvement of the posterior part of the right DLPFC during encoding than during recognition. This posterior dorsal prefrontal area, however, should be distinguished from more anterior right prefrontal areas also included in HERA. The areas included are centered in the anterior part of the middle frontal gyrus at the junction of BA 46/9/10. They are commonly recruited across various episodic retrieval studies (Buckner et al. 1996b; Haxby et al. 1996; Wagner et al. 1998a; see also Lepage et al. 2000). Typically, this latter region is activated independent of the task demands and the material to be retrieved. There is an ongoing debate about the functional role of this brain region, either reflecting retrieval effort, retrieval success, or retrieval monitoring (Rugg et al. 1996; Wilding and Rugg 1996; Buckner et al. 1998b; Henson et al. 1999). In this study we did not find a similar brain response, even when considering a delayed lag of the hemodynamic response function (as proposed by Schacter et al. $1997^{1}$ ). Thus, the view that the brain re-

\footnotetext{
${ }^{1}$ Based on the findings of Schacter et al. who reported a late onset of the right anterior PFC activity we analyzed the present data with a delay of $6 \mathrm{sec}$ instead of $4 \mathrm{sec}$. We observed a strong contribution of the superior cerebral veins to the BOLD response in this time range. As noted by Schacter such a hemodynamic factor may also account for the late onset of his right anterior prefrontal activity.
} 
Table 3. Encoding Phase: Brain Activation for Identifiable and Nonidentifiable Novel Sounds

\begin{tabular}{|c|c|c|c|c|c|c|c|c|}
\hline & \multicolumn{4}{|c|}{ Identifiable novels ${ }^{\mathrm{a}}$} & \multicolumn{4}{|c|}{ Nonidentifiable novels ${ }^{\mathrm{a}}$} \\
\hline & \multicolumn{2}{|c|}{ verbal task } & \multicolumn{2}{|c|}{ nonverbal task } & \multicolumn{2}{|c|}{ verbal task } & \multicolumn{2}{|c|}{ nonverbal task } \\
\hline & (LH) & $(\mathrm{RH})$ & $(\mathrm{LH})$ & $(\mathrm{RH})$ & (LH) & $(\mathrm{RH})$ & $(\mathrm{LH})$ & $(\mathrm{RH})$ \\
\hline Anterior insula & $\begin{array}{c}3.48 \\
(0.36)\end{array}$ & $\begin{array}{c}3.75 \\
(0.49)\end{array}$ & $\begin{array}{c}3.70 \\
(0.33)\end{array}$ & $\begin{array}{c}3.51 \\
(0.49)\end{array}$ & $\begin{array}{c}3.81 \\
(0.78)\end{array}$ & $\begin{array}{c}4.38 \\
(0.72)\end{array}$ & $\begin{array}{c}4.63 \\
(0.69)\end{array}$ & $\begin{array}{c}4.42 \\
(0.60)\end{array}$ \\
\hline Thalamus & $\begin{array}{c}2.18 \\
(0.56)\end{array}$ & $\begin{array}{c}2.34 \\
(0.44)\end{array}$ & $\begin{array}{c}2.13 \\
(0.53)\end{array}$ & $\begin{array}{c}2.27 \\
(0.48)\end{array}$ & $\begin{array}{c}2.04 \\
(0.50)\end{array}$ & $\begin{array}{c}2.18 \\
(0.68)\end{array}$ & $\begin{array}{c}2.67 \\
(0.40)\end{array}$ & $\begin{array}{c}2.16 \\
(0.54)\end{array}$ \\
\hline Superior temporal plane & $\begin{array}{c}3.53 \\
(0.40)\end{array}$ & $\begin{array}{c}3.40 \\
(0.42)\end{array}$ & $\begin{array}{c}3.65 \\
(0.46)\end{array}$ & $\begin{array}{c}3.01 \\
(0.59)\end{array}$ & $\begin{array}{c}2.39 \\
(0.56)\end{array}$ & $\begin{array}{c}3.71 \\
(0.63)\end{array}$ & $\begin{array}{c}3.52 \\
(0.62)\end{array}$ & $\begin{array}{c}2.60 \\
(0.62)\end{array}$ \\
\hline
\end{tabular}

${ }^{\mathrm{a}}(\mathrm{LH})$ Left hemisphere; $(\mathrm{RH})$ right hemisphere.

Mean z-scores and S.E.M.S across all subjects. No specific lateralization of brain activity was observed for any of these regions across encoding tasks.

sponse at the junction of BA 49/9/10 was delayed temporally with respect to that in more posterior prefrontal areas cannot account for the absence of a right anterior prefrontal brain response in the present study.

Another possible account for the absence of such an activity may be derived from the view that the engagement of right anterior prefrontal depends on the context in which retrieval is conducted. Wagner et al. (1998a) reported right anterior PFC activation to be influenced by task instructions with all other test parameters being constant. It is conceivable that differences in instructions cause differences in the cognitive state underlying an established retrieval strategy. In addition, retrieval strategies may also be induced when task demands are constant for repetitive executions as it is usually the case in block designs. Thus, comparison across blocks would reveal differences in retrieval strategy and thereby in right anterior PFC activation. In this study we employed an event related analysis and contrasted the BOLD response to stimuli of interest with a baseline within the same test period, a comparison that might have reduced possible effects of retrieval strategy. This view could partially account for the apparent inconsistency between the present data and the HERA model. HERA was articulated based on the results of PET studies that examine retrieval-related brain activation pattern in block designs. This could have overemphasized the contribution of con- text dependent retrieval strategies to right prefrontal cortex activation in HERA. In contrast, this study focused on more transient, content-specific effects that are reliant on posterior dorsolateral prefrontal areas. Thus, conflicting results across different studies might be reconciled if a functional distinction between anterior and posterior prefrontal activation sites is considered.

\section{Activation in Other Brain Areas}

The observation of bilateral activation of the anterior insula is similar to previous findings reported by Buckner and colleagues (1996b, 1998b) using word recognition tasks. In their neuroimaging studies they observed significant blood flow changes in regions of the anterior insula near the frontal operculum as a function of retrieval effort induced by the depth of encoding and they inferred that these regions might be sensitive to overall task effort. They argued further that the effort-related modulation may reflect the reduced time on task achieved because of deep encoding at the time of study. In this study the variation of the encoding instructions did not result in significant differences in the recognition performance. This may be taken as an indication that the effort imposed by both tasks was quite similar and did not lead to a differential activation of the anterior insula.

An alternative interpretation is provided by the conditioning literature. Several studies have demonstrated that

Table 4. Coefficients of Kendall's Correlations of the fMRI Activation in the Left and Right Hemispheres During Encoding and Memory Performance for Novel Sounds

\begin{tabular}{|c|c|c|c|c|c|c|c|}
\hline \multicolumn{4}{|c|}{ Verbal encoding task } & \multicolumn{4}{|c|}{ Nonverbal encoding task } \\
\hline \multicolumn{2}{|c|}{ identifiable novels } & \multicolumn{2}{|c|}{ nonidentifiable novels } & \multicolumn{2}{|c|}{ identifiable novels } & \multicolumn{2}{|c|}{ nonidentifiable novels } \\
\hline (LH) & $(\mathrm{RH})$ & $(\mathrm{LH})$ & $(\mathrm{RH})$ & $(\mathrm{LH})$ & $(\mathrm{RH})$ & $(\mathrm{LH})$ & $(\mathrm{RH})$ \\
\hline $0.59 *$ & 0.22 & 0.14 & -0.21 & 0.36 & $0.50^{* *}$ & $-0.54 * *$ & 0.18 \\
\hline
\end{tabular}

(*) $P<0.05 ;(*) P<0.10$.

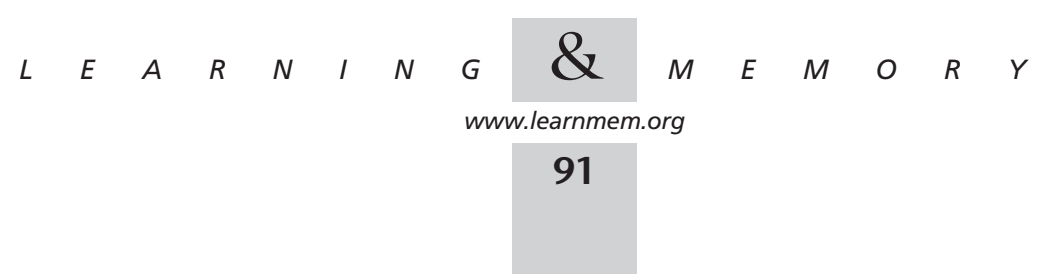



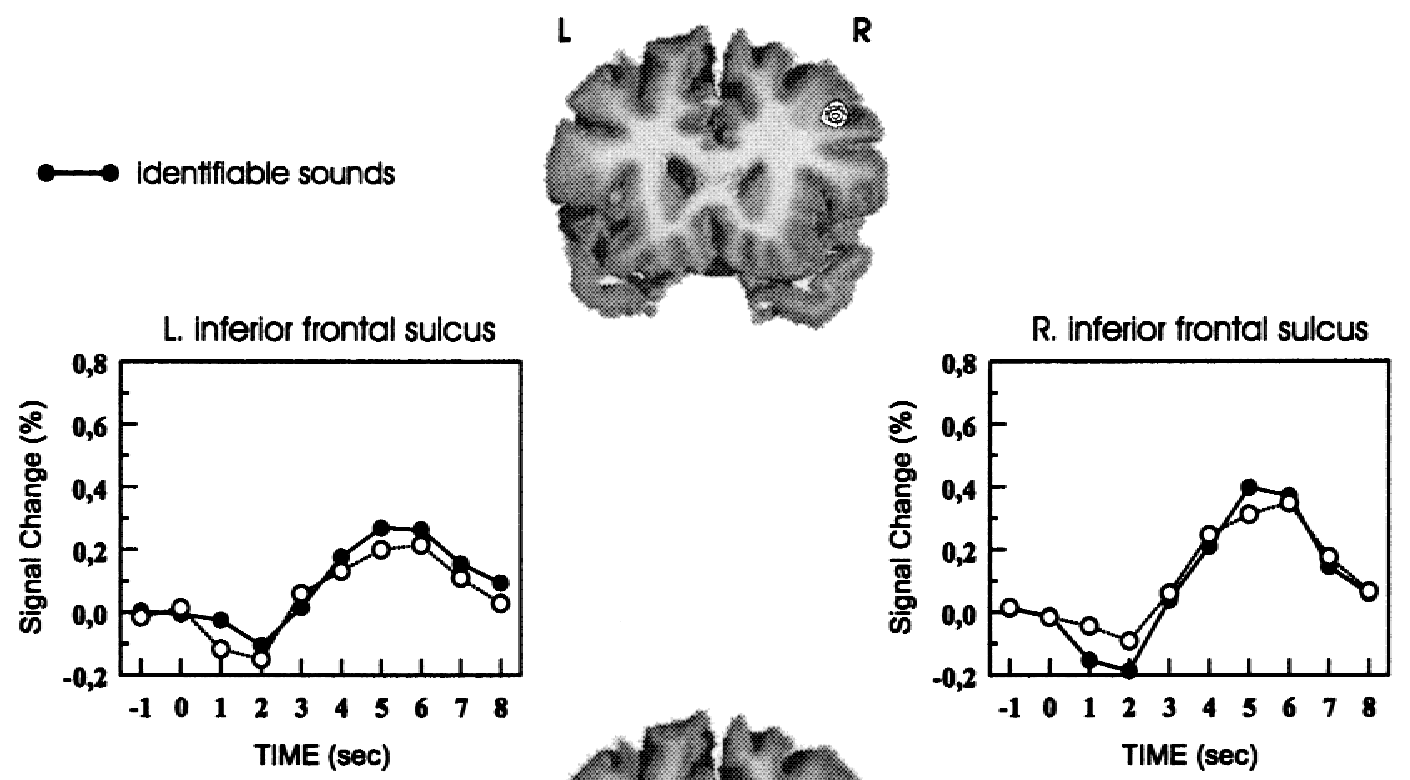

O-O non identifiable sounds

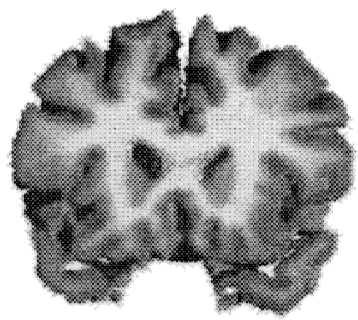

TIME (sec)

Figure 4 Recognition test nonverbal task. Dorsolateral prefrontal cortex activation for identifiable (top, $\bullet$ ) and nonidentifiable (bottom, $\bigcirc)$ novel sounds. Functional images were superimposed on an individual brain in Talairach space. The images were thresholded at $z=2.33$ $(P<0.01$, one tailed). For details, see Fig. 1 . Peak activations were located in right $(45,9,30)$ dorsolateral prefrontal cortex for identifiable novels.

the insular cortex plays an important role in different aversive conditioning tasks (Kosslyn et al. 1996; Büchel et al. 1998). Büchel et al. (1998) using fMRI reported an increased BOLD response in the anterior insula in response to stimuli conditioned with an aversive tone of $\sim 100 \mathrm{~dB}$, even when they were not paired with the aversive stimulus. Furthermore, these stimuli elicited an autonomic skin conductance response similar to that observed for auditory novel stimuli (Knight 1996). In light of these findings one could argue that the novel sounds, because they were presented at $95 \mathrm{~dB}$, act as aversive stimuli and evoke autonomic responses integrated by the anterior insula (for an overview of insular functions, see Flynn et al. 1999).

Another region that exhibited greater response to novel stimuli as compared to tones was the bilateral superior temporal plane. This activation was restricted to the encoding conditions and therefore might reflect encodingrelated activity. However, the absence of superior temporal activity in the recognition phase, that is, the second presentation of the sounds, point to a specific involvement of this brain region in novelty detection. This latter view is supported by the fact that no memory-related modulations of the activity of the superior temporal plane were observed in the present study. Further support for this novelty detection account is provided by a recent study showing bilateral activation of the superior temporal gyrus to novel sounds in an auditory classification task (Opitz et al. 1999). In this latter study superior temporal gyrus activation was directly related to processes that accompany the involuntary shift of attention toward such stimuli (Knight 1996; Näätänen 1992).

\section{Conclusion}

The present results together with previous findings suggest a content-specific rather than process-specific lateralization of the activity in the posterior dorsolateral prefrontal cortex in an explicit memory task. The observed brain activations were modulated by task instructions and stimulus properties in a complex manner. The availability of verbal codes for identifiable stimuli during encoding does not necessarily imply the use of these codes during recognition. Rather, our results point to the importance of encoding instructions in determining the use of content-specific, that is, verbal or nonverbal, strategies in memory tasks and the respective lateralization of the prefrontal cortex.

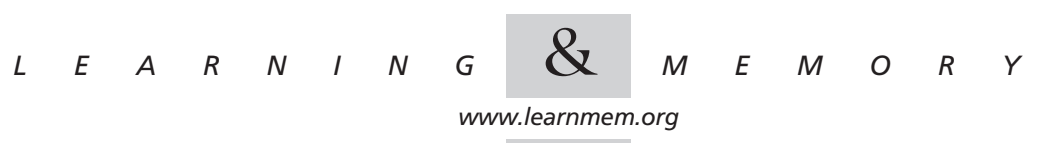




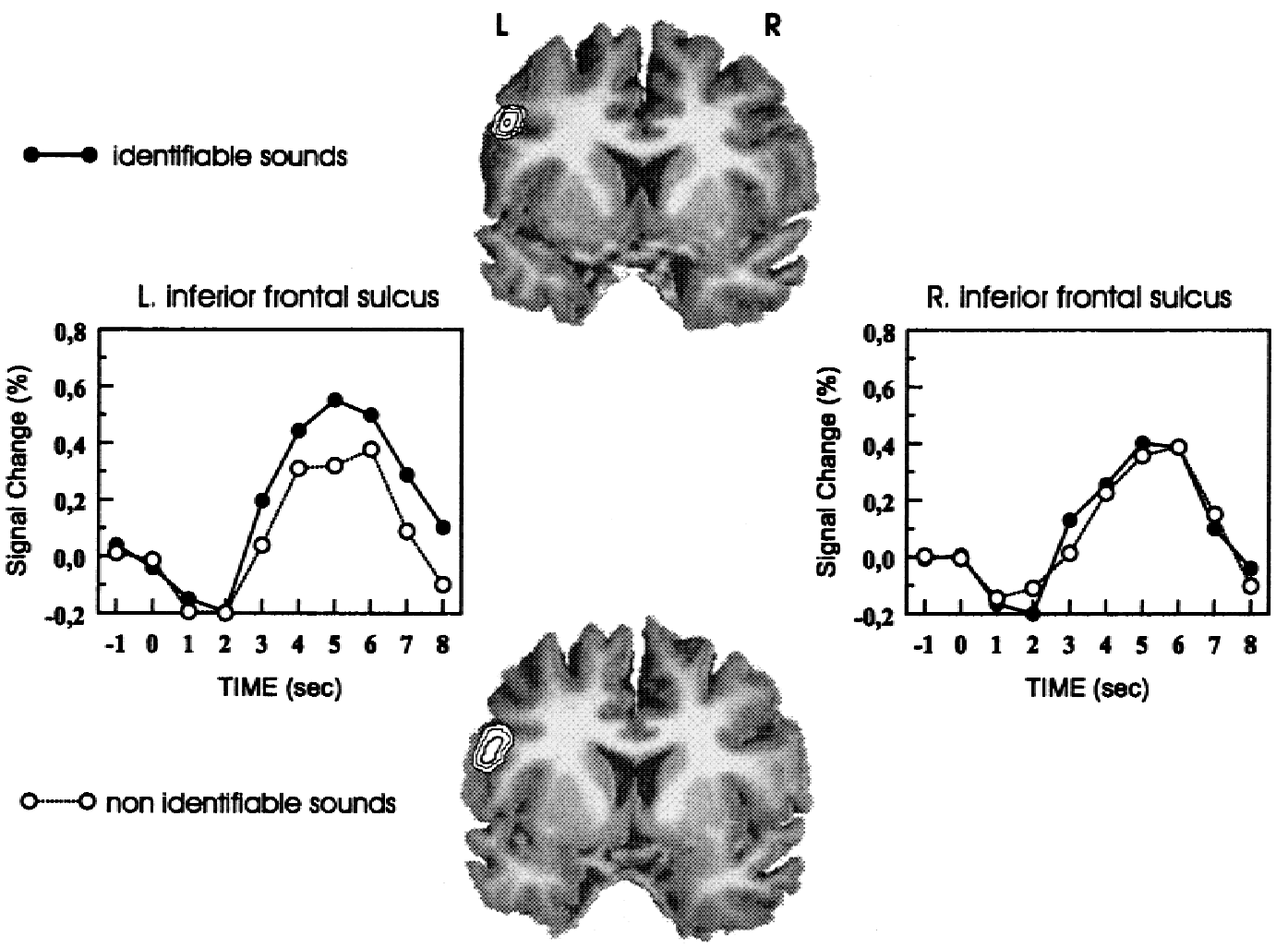

Figure 5 Recognition test verbal task. Dorsolateral prefrontal cortex activation for identifiable (top, $\bullet$ ) and nonidentifiable $($ bottom, $\bigcirc)$ novel sounds. Peak activations were located in left $(-46,6,33$ and $-48,8,24)$ dorsolateral prefrontal cortex for identifiable novels and nonidentifiable novels, respectively. (For details, see Fig. 4.)

\section{METHODS}

\section{Subjects}

Twenty right-handed volunteers between the ages of 20 and 29 years $(9$ male) participated in the study. All subjects had given informed consent prior to participation. Because of coarse movement artifacts in the fMRI session, two subjects (one of each encoding task group) had to be excluded from all analyses.

\section{Stimuli}

The stimuli used in this study were pure sine tones and unique environmental sounds. The frequency of the tones was $600 \mathrm{~Hz}$. The environmental sounds (novels) were divided into two groups: identifiable novel sounds and nonidentifiable novels. This separation was based on a pilot study (described in Mecklinger et al. 1997) in which 15 subjects that did not participate in this study were asked (1) to write down the first word that came to mind after hearing the sound, and (2) to indicate how certain they were about their judgment on a four-point rating scale ranging from very uncertain (0) to very certain (3). Based on this rating two groups of 55 novels each were selected. All stimuli had a duration of $200 \mathrm{msec}$ (including 10-msec rise and 40-msec fall time). The stimuli were delivered to the subjects using nonmagnetic air-conducting headphones.

\section{Behavioral Procedure}

The experiment consisted of an encoding phase followed by a recognition phase. In the encoding phase subjects listened to 320 tones and 40 identifiable and 40 nonidentifiable novel stimuli. Each novel stimulus was randomly selected from the respective set of 55 novel stimuli and occurred only once in the encoding phase. The interstimulus interval was two sec. To keep the subjects attention on the task the length of an individual trial (i.e., the interval between two consecutive novels) varied randomly between 8 and 12 seconds. This spaced presentation of novel stimuli allowed an event-related analysis of the hemodynamic brain responses (Buckner et al. 1996a; Pollmann et al. 1998).

Half of the participants were instructed to judge whether or not a novel sound was louder as the preceding pure tones-a nonverbal task. The other half of the subjects were asked to judge whether or not a novel sound could be unambiguously verbally described (e.g., dog barking, telephone bell)-a verbal task. Subjects in both groups were asked to try to remember the novel sounds for a later memory test.

The recognition test consisted of the 80 previously heard sounds and 30 new sounds presented randomly intermixed and separated by the pure tones. As during encoding the interval between two successive novel stimuli varied randomly between 8 and 12 sec. Participants were instructed to determine whether each presented novel stimulus had been studied previously or not. All responses in the encoding and recognition phases were indicated by key presses with the index or the middle finger of the right hand.

The efficiency of the behavioral manipulations was pretested in a pilot study examining sixteen subjects that did not participate

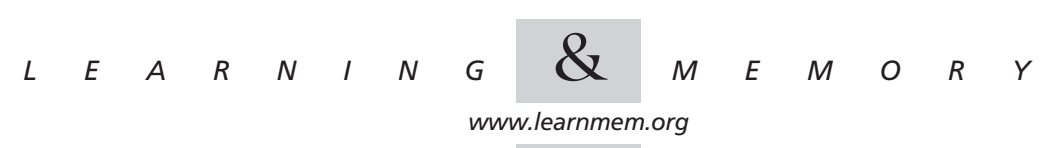



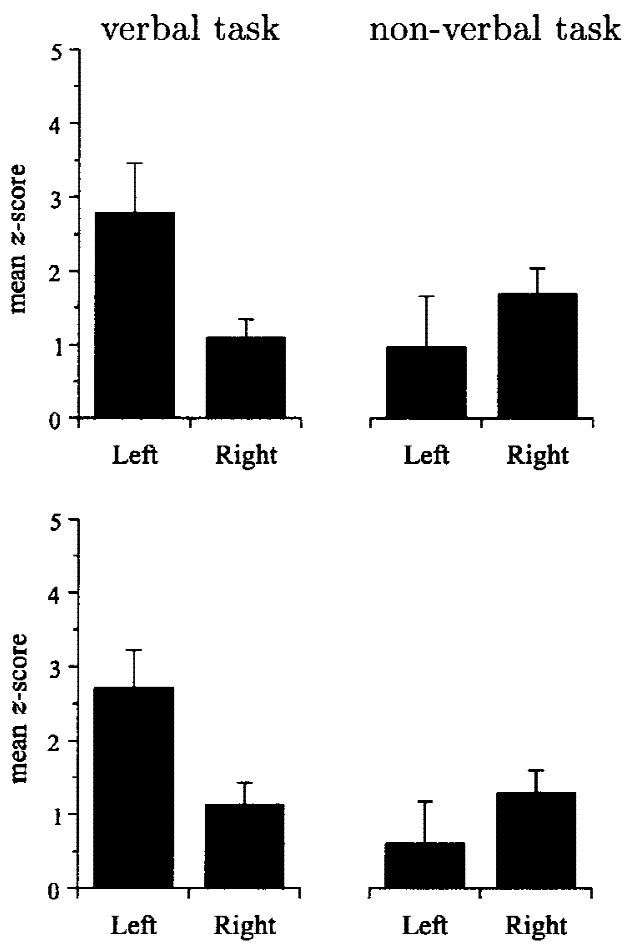

Figure 6 Recognition phase. Mean activation $z$-values and standard error of means of the regions depicted in Figs. 4 and 5. (Top) Identifiable novels; (bottom) nonidentifiable novels.

in the present experiment. More than $80 \%$ of the subjects showed a reliable recognition performance following intentional encoding. This was in agreement with previous results (Cycowicz and Friedman 1999) that showed robust memory effects for novel sounds only following intentional but not following incidental learning. For this reason we used an intentional encoding instruction along with the verbal/nonverbal tasks.

\section{MR Procedures}

Imaging was performed with a 3T Bruker Medspec 30/100 system. The standard birdcage head coil was used. Subjects were supine on the scanner bed, with a stereotactic fixation system used to reduce head motion. In a separate session high resolution whole-brain images were acquired to assist localization of activation foci using a $T_{1}$ weighted three-dimensional segmented MDEFT (128 slice sagittal, $1.5 \mathrm{~mm}$ thickness, $256 \times 256$ pixel matrix). For each subject, structural and functional (echo-planar) images were collected within a $1.5 \mathrm{hr}$ session. Conventional $T_{1}$ weighted anatomic images (IR-RARE sequence: $\mathrm{TE}=20 \mathrm{msec}, \mathrm{TR}=3750 \mathrm{msec}$, in-plane resolution $0.325 \mathrm{~mm}^{2}$ ) in plane with the echo-planar images were acquired to align the functional images to the three-dimensional images. Finally, functional images were recorded in two separate runs for encoding and recognition using a gradient EPI sequence ( $\mathrm{TE}=40 \mathrm{msec}, \mathrm{TR}=1000 \mathrm{msec}$ ) sensitive to BOLD contrast. An acquisition volume consisted of eight axial slices, parallel to the plane intersecting the anterior and posterior commissures (AC-PC plane), with a $3-\mathrm{mm}^{2}$ in-plane and a $7-\mathrm{mm}$ through-plane resolution. The most inferior slice was positioned $15 \mathrm{~mm}$ below the AC-PC plane. Four discarded volumes were acquired at the beginning of each run while tones were presented to allow stabilization of mag- netization. Thus, a total of 804 and 1000 volumes were collected during the encoding and recognition phase, respectively.

\section{Data Analysis}

fMRI data were processed using the Brian software package (Kruggel and Lohmann 1996). Prior to statistical analyses, motions artifacts were corrected using an affine rotation and translation correction (Kruggel et al. 1998). Second, low-frequency signal fluctuations were removed on a voxel-by-voxel basis (Kruggel et al. 1998). Finally, a spatial smooth with a Gaussian kernel of two voxels was applied to emphasize spatially coherent activation pattern. For each subject the fMRI time series from each voxel were correlated with a reference waveform. This reference wave form resembled the time course of the shortest trial $(8 \mathrm{sec})$. This time period was chosen to ensure equal contribution of trials with different length to the hemodynamic response function. To account for the physiological delay in the hemodynamic response the reference waveform was shifted by $4 \mathrm{sec}$ (Buckner et al. 1998b; Malonek and Grinvald 1995).

The activation map revealed event-related activity evoked by novel sounds relative to pure tones. All novel sounds from the encoding phase and correctly recognized novel sounds from the recognition phase entered this analysis. Activation maps were registered with individual high resolution three-dimensional data sets and transformed into stereotactic Talairach space (Friston et al. 1995; Kruggel 1995). Multi-subject averaging was used to determine the presence of significantly activated brain regions (Bosch 2000).

To analyze interhemispheric differences of significant BOLD signal across subjects the center of fMRI activation were identified within regions reflecting the summed activity collapsed across tasks and novel types separately for encoding and recognition. Spherical regions (4-mm radius) were defined around each of these peak activations, and mean $z$-scores ${ }^{2}$ for each region were then computed separate for each condition (Bosch 2000). Mean $z$-scores were subjected to repeated-measure ANOVAs with the betweensubject factor task ( 2 levels), and two within-subject factors hemisphere (2 levels) and novel type (2 levels).

A second analysis was based on a Kendall's rank-order correlation of subsequent recognition performance and the encoding related brain activity to the novel sounds. Again spherical regions were used to determine encoding related brain activity. The mean $z$-scores were correlated with a measure of pure recognition, $\operatorname{Pr}$ (i.e., the difference between the hit rate and the false alarm rate, Snodgrass and Corwin 1988) across subjects. This procedure was performed to identify brain regions whose activity reliably predicted memory performance for novel sounds.

\section{ACKNOWLEDGMENTS}

This work was supported by the Leibniz-Prize of the German Research Foundation awarded to A.D.

The publication costs of this article were defrayed in part by payment of page charges. This article must therefore be hereby

${ }^{2}$ To account for inter individual differences in the hemodynamic response not related to the investigated manipulation $z$-scores were used for statistical analyses instead of contrasts. The $z$-score, i.e., a contrast of a voxel weighted by its noise, provides a possibility for a comparison of the activations across subjects according to the respective signal to noise ratio.

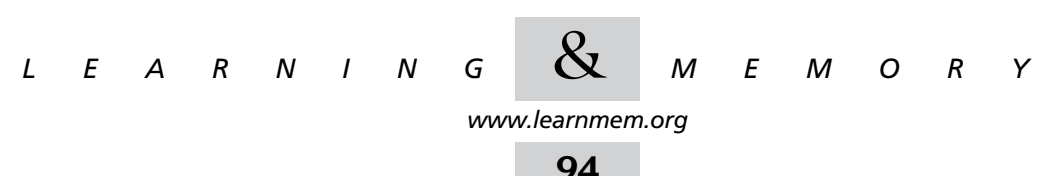


Table 5. Recognition Test: Brain Activation for Identifiable and Nonidentifiable Novel Sounds

\begin{tabular}{|c|c|c|c|c|c|c|c|c|}
\hline & \multicolumn{4}{|c|}{ Identifial novels } & \multicolumn{4}{|c|}{ Nonidentifiable novels } \\
\hline & \multicolumn{2}{|c|}{ verbal task } & \multicolumn{2}{|c|}{ nonverbal task } & \multicolumn{2}{|c|}{ verbal task } & \multicolumn{2}{|c|}{ nonverbal task } \\
\hline & (LH) & $(\mathrm{RH})$ & (LH) & $(\mathrm{RH})$ & $(\mathrm{LH})$ & $(\mathrm{RH})$ & (LH) & $(\mathrm{RH})$ \\
\hline Anterior insula & $\begin{array}{c}2.12 \\
(0.45)\end{array}$ & $\begin{array}{c}1.16 \\
(0.37)\end{array}$ & $\begin{array}{c}2.32 \\
(0.69)\end{array}$ & $\begin{array}{c}1.74 \\
(0.55)\end{array}$ & $\begin{array}{c}2.30 \\
(0.46)\end{array}$ & $\begin{array}{c}1.48 \\
(0.40)\end{array}$ & $\begin{array}{c}1.61 \\
(0.65)\end{array}$ & $\begin{array}{c}1.38 \\
(0.68)\end{array}$ \\
\hline Thalamus & $\begin{array}{c}2.40 \\
(0.50)\end{array}$ & $\begin{array}{c}2.81 \\
(0.68)\end{array}$ & $\begin{array}{c}1.88 \\
(0.56)\end{array}$ & $\begin{array}{c}2.66 \\
(0.44)\end{array}$ & $\begin{array}{c}2.67 \\
(0.40)\end{array}$ & $\begin{array}{c}3.16 \\
(0.54)\end{array}$ & $\begin{array}{c}2.25 \\
(0.53)\end{array}$ & $\begin{array}{c}2.17 \\
(0.48)\end{array}$ \\
\hline
\end{tabular}

Mean z-scores and S.E.M. across all subjects. No specific lateralization of brain activity was observed for any of these regions across encoding tasks.

marked "advertisement" in accordance with 18 USC section 1734 solely to indicate this fact.

\section{REFERENCES}

Bosch, V. 2000. Statistical analysis of multi-subject fMRI data: The assessment of focal activations. J. Mag. Res. Imag. 11: 61-64.

Brewer, J.B., Z. Zhao, J.E. Desmond, G.H. Glover, and J.D.E. Gabrieli. 1998. Making memories: Brain activity that predicts how well visual experience will be remembered. Science 281: 1185-1187.

Büchel, C., J. Morris, R.J. Dolan, and K.J. Friston. 1998. Brain systems mediating aversive conditioning: An event-related fMRI study. Neuron 20: $947-957$.

Buckner, R.L., P.A. Bandettini, K.M. Craven, R.L. Savoy, S.E. Petersen, M.E Raichle, and B.R. Rosen. 1996a. Detection of cortical activation during averaged single trials of a cognitive task using functional magnetic resonance imaging. Proc. Natl. Acad. Sci. 93: 14878-14883.

Buckner, R.L., M.E. Raichle, F.M. Miezin, and S.E. Petersen. 1996b. Functional anatomic studies of memory retrieval for auditory words and visual pictures . J. Neurosci. 16: 6219-6235.

Buckner, R.L., W. Koutstaal, D. Schacter, A. Dale, M. Rotte, and B. Rosen 1998a. Functional-anatomic study of episodic retrieval using fMRI: II. Selective averaging of event-related fMRI trials to test the retrieval success hypothesis. Neuroimage 7: 163-175.

Buckner, R.L., W. Koutstaal, D. Schacter, A. Wagner, and B. Rosen. 1998b. Functional-anatomic study of episodic retrieval using fMRI: I. Retrieval effort versus retrieval success. Neuroimage 7: 151-162.

Cycowicz, Y.M. and D. Friedman. 1999. The effect of intention to learn novel, environmental sounds on the novelty $\mathrm{P} 3$ and old/new recognition memory. Biol. Psych. 50: 35-60.

Dolan, R.J. and P.C. Fletcher. 1997. Dissociating prefrontal and hippocampal function in episodic memory encoding. Nature 388: $582-585$.

Flynn, F.G., D.F. Benson, and A. Ardila. 1999. Anatomy of the insula-functional and clinical correlates. Aphasiology 13: 55-78.

Friston, K.J., J. Ashburner, C.D. Frith, J.B. Poline, J.D. Heather, and R.S.J Frackowiak. 1995. Spatial registration and normalization of images. Hum. Brain Mapping 2: 165-189.

Gazzaniga, M., and C.S. Smylie. 1983. Facial recognition and brain asymmetries: Clues to underlying mechanisms. Ann. Neurol. 13: 536-540.

Haxby, J.V., L.G. Ungerleider, B. Horwitz, S.I. Rapoport, and C.L. Grady. 1995. Hemispheric differences in neural systems for face working memory: A PET-rCBF study. Hum. Brain Mapping 3: 68-82.

Haxby, J.V., L.G. Ungerleider, B. Horwitz, J.M. Maisog, S.I. Rapoport, and C.L. Grady, 1996. Face encoding and recognition in the human brain. Proc. Natl. Acad. Sci. 93: 922-927.

Henson, R.N., M.D. Rugg, T. Shallice, O. Josephs, and R.J. Dolan. 1999. Recollection and familiarity in recognition memory: An event-related functional magnetic resonance imaging study. J. Neurosci. 19: 3962-3972
Kelley, W.M., R.L. Buckner, and S.E. Petersen. 1998a. Asymmetric frontal activation during episodic memory: What kind of specificity? Response. Trends Cogn. Sci. 2: 421.

Kelley, W.M., F.M. Miezin, K.B. McDermott, R.L. Buckner, M.E. Raichle, N.J. Cohen, A.Z. Snyder, and S.E. Petersen. 1998b. Hemispheric specialization in human dorsal frontal cortex and medial temporal lobe for verbal and nonverbal memory encoding. Neuron 20: 927-936.

Klingenberg, T., and P.E. Roland. 1998. Right prefrontal activation during encoding, but not during retrieval, in a non-verbal paired-associates task. Cereb. Cortex 8: 73-79.

Knight, R.T. 1996. Contribution of human hippocampal region to novelty detection. Nature 383: 256-259.

Kosslyn, S.M., L.M. Shin, W.L. Thompson, R.J. Mcnally, S.L. Rauch, R.K Pitman, and N.M. Alpert. 1996. Neural effects of visualizing and perceiving aversive stimuli: A PET investigation. NeuroReport 7: 1569-1576

Kruggel, F. 1995. Automatical registration of brain volume dataset. In Information processing in medical imaging (ed. N. Ayehe), pp. 387-412. Kluwer, Amsterdam, The Netherlands.

Kruggel, F. and G. Lohmann. 1996. BRIAN (Brain Image Analysis) - A toolkit for the analysis of multimodal brain datasets. In $C A R^{\prime} 96$ Computer Assisted Radiology, Paris 1996 (ed. H.U. Lemke, M.W Vannier, K. Inamura, and A.G. Farman), pp. 323-328. Elsevier, Amsterdam, The Netherlands.

Kruggel, F., X. Descombes, and D.Y. von Cramon. 1998. Preprocessing of fMRI datasets. In Workshop on biomedical image analysis (ed. B. Vemuri), pp. 323-330. IEEE Press, Los Angeles, CA.

Kutas, M. and S.A. Hillyard. 1983. Event-related brain potentials to grammatical errors and semantic anomalies. Mem. Cogn. 11: 539-550.

Lepage, M., O. Ghaffar, L. Nyberg, and E. Tulving. 2000. Prefrontal cortex and episodic memory retrieval mode. Proc. Natl. Acad. Sci. 97: 506-511.

Malonek, D. and A. Grinvald. 1995. Interactions between electrical activity and cortical microcirculation revealed by imaging spectroscopy: Implications for functional brain mapping. Science 272: 551-554.

McDermott, K.B., R.L. Buckner, S.E. Petersen, W.M. Kelley, and A.L. Sanders. 1999. Set- and code-specific activation in the frontal cortex: An fMRI study of encoding and retrieval of faces and words. J. Cogn. Neurosci. 11: 631-640.

Mecklinger, A., B. Opitz, and A.D. Friederici. 1997. Semantic aspects of novelty detection in humans. Neurosci. Lett. 235: 65-68.

Milner, B. and L. Taylor. 1972. Right hemisphere superiority in tactile pattern recognition after cerebral commissurotomy: Evidence for nonverbal memory. Neuropsychologia 10: 1-15.

Näätänen, R. 1992. Attention and brain function. Erlbaum, Hillsdale, NJ.

Nyberg, L., R. Cabeza, and E. Tulving. 1996. PET studies of encoding and retrieval: The HERA model. Psych. Bull. and Rev. 3: 135-148.

- 1998. Asymmetric frontal activation during episodic memory: What kind of specificity? Trends Cognit. Sci. 2: 419-420.

Opitz, B., A. Mecklinger, A.D. Friederici, and D.Y. von Cramon. 1999. The

\section{$\begin{array}{lllllllllllllllllllllllllll} & E & A & R & N & I & N & G & \mathbf{Z} & M & E & M & O & R & Y\end{array}$


functional neuroanatomy of novelty processing: Integrating ERP and fMRI results. Cereb. Cortex 9: 379-391.

Pigott, S. and B. Milner. 1993. Memory for different aspects of complex visual scenes after unilateral temporal- or frontal-lobe resection. Neuropsychologia 31: 1-15.

Pollmann, S., C.J. Wiggins, D.G. Norris, D.Y. von Cramon, and T. Schubert. 1998. Use of short intertrial intervals in single-trial experiments: A 3T fMRI-study. Neuroimage 8: 327-339.

Rugg, M.D., P.C. Fletcher, C.D. Frith, R.S.J. Frackowiak, and R.J. Dolan. 1996. Differential response of the prefrontal cortex in successful and unsuccessful memory retrieval. Brain 119: 2073-2083.

Schacter, D.L., R.L. Buckner, W. Koutstaal, A.M. Dale, and B.R. Rosen. 1997. Late onset of anterior prefrontal activity during true and false recognition: An event-related fMRI study. Neuroimage 6: 259-260.

Snodgrass, J.C. and J. Corwin. 1988. Pragmatics of measuring recognition memory: Applications to dementia and amnesia. J. Exp. Psych. 117: 34-50.

Talairach, J. and P. Tournoux. 1988. Co-planar stereotaxis atlas of the buman brain. Thieme, New York, NY.

Tulving, E., S. Kapur, F.I.M. Craik, M. Moscovitch. and S. Houle. 1994 Hemispheric encoding/retrieval asymmetry in episodic memory:
Positron emission tomography findings. Proc. Natl. Acad. Sci. 91: 2016-2020.

Wagner, A.D., J.E. Desmond, G.H. Glover, and J.D. Gabrieli. 1998a. Prefrontal cortex and recognition memory: Functional MRI evidence for context-dependent retrieval processes. Brain 281: 1188-1190.

Wagner, A.D., R.A. Poldrack, L.L. Eldridge, J.E. Desmond, G.H. Glover, and J.D. Gabrieli. 1998b. Material-specific lateralization of prefrontal activation during episodic encoding and retrieval. NeuroReport 9: 3711-3717.

Wagner, A.D., D.L. Schacter, M. Rotte, W. Koutstaal, A. Maril, A.M. Dale, B.R. Rosen, and R.L. Buckner. 1998c. Building memories: Remembering and forgetting of verbal experiences as predicted by brain activity.Science 281: 1188-1190.

Wilding, E.L. and M.D. Rugg. 1996. An event-related potential study of recognition memory with and without retrieval source. Brain 119: 889-905.

Zatorre, R.J., A.C. Evans, and E. Meyer. 1994. Neural mechanisms underlying melodic perception and memory for pitch. J. Neurosci. 14: 1908-1919.

Received October 27, 1999; accepted in revised form February 7, 2000. 


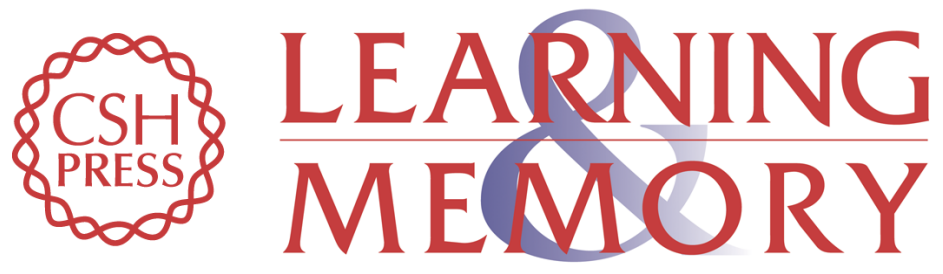

\section{Functional Asymmetry of Human Prefrontal Cortex: Encoding and Retrieval of Verbally and Nonverbally Coded Information}

Bertram Opitz, Axel Mecklinger and Angela D. Friederici

Learn. Mem. 2000, 7:

Access the most recent version at doi:10.1101//m.7.2.85

References This article cites 35 articles, 10 of which can be accessed free at: http://learnmem.cshlp.org/content/7/2/85.full.html\#ref-list-1

License

Email Alerting Receive free email alerts when new articles cite this article - sign up in the box at the Service top right corner of the article or click here. 